Supporting Information

\title{
Aptamer displacement reaction from live-cell surfaces and its applications
}

Long Li ${ }^{1}$, Xigao Chen ${ }^{1}$, Cheng Cui ${ }^{1,2,3}$, Xiaoshu Pan ${ }^{1}$, Xiaowei $\mathrm{Li}^{1}$, Hoda Safari Yazd ${ }^{1}$, Qiong $\mathrm{Wu}^{1}$, Liping $\mathrm{Qiu}^{2}$, Juan $\mathrm{Li}^{2,3,4 *}$, Weihong Tan*1,2

${ }^{1}$ Department of Chemistry, Department of Physiology and Functional Genomics, UF Health Cancer Center, UF Genetics Institute, University of Florida, Gainesville, Florida 32611, USA

${ }^{2}$ Molecular Science and Biomedicine Laboratory, State Key Laboratory of Chemo/Biosensing and Chemometrics, College of Chemistry and Chemical Engineering, College of Biology, Collaborative Innovation Center for Chemistry and Molecular Medicine, Hunan University, Changsha 410082, China

${ }^{3}$ Institute of Molecular Medicine (IMM), Renji Hospital, Shanghai Jiao Tong University School of Medicine, and College of Chemistry and Chemical Engineering, Shanghai Jiao Tong University, Shanghai 200240, China.

${ }^{4}$ Institute of Cancer and Basic Medicine (IBMC), Chinese Academy of Sciences; The Cancer Hospital of the University of Chinese Academy of Sciences, Hangzhou, Zhejiang 310022, China.

Corresponding author's E-mail: tan@chem.ufl.edu; juanli@sjtu.edu.cn 


\section{Supplementary Discussion1}

The binding affinity of PTK7/aptamer $(\mathrm{Kd}=0.80 \mathrm{nM})$ is much higher than $\mathrm{SSB} /$ single-stranded DNA aptamer $(\mathrm{Kd}=30 \mathrm{nM})$. However, the equilibrium state of those molecules depends not only on their binding affinities but also on their relative concentrations. In our system, the process of the aptamer displacement reaction can be written as:

$$
\text { SSB }+ \text { PTK7 } \cdot \text { Sgc8 } \rightarrow \text { SSB } \cdot \text { Sgc8 }+ \text { PTK7 }
$$

It can also be written as a two-step reaction:

$$
\begin{gathered}
\text { PTK7 } \cdot \text { Sgc8 } \leftrightarrow \text { PTK7 + Sgc8 } \\
\text { SSB + Sgc8 } 4 \text { SSB } \cdot \text { Sgc } 8
\end{gathered}
$$

Since a large excess of SSB is introduced to bind Sgc8, the reaction equilibrium moves to the right, indicating that more free Sgc8 would be generated and would bind with SSB in the second reaction.

The dissociated constant can be written as:

$$
\begin{gathered}
\mathrm{Kd} 1=\frac{[\mathrm{PTK} 7][\mathrm{Sgc} 8]}{[\mathrm{PTK} 7 \cdot \operatorname{Sgc} 8]} \\
\mathrm{Kd} 2=\frac{[\mathrm{SSB}][\mathrm{Sgc} 8]}{[\mathrm{SSB} \cdot \mathrm{Sgc} 8]}
\end{gathered}
$$

Which can also be expressed as:

$$
\begin{aligned}
{[\mathrm{PTK} 7 \cdot \mathrm{Sgc} 8] } & =\frac{[\mathrm{PTK} 7][\mathrm{Sgc} 8]}{\mathrm{Kd} 1} \\
{[\mathrm{SSB} \cdot \mathrm{Sgc} 8] } & =\frac{[\mathrm{SSB}][\mathrm{Sgc} 8]}{\mathrm{Kd} 2}
\end{aligned}
$$

The ratio $(\mathrm{F})$ of $\mathrm{PTK} \cdot \mathrm{Sgc} 8$ and total $\mathrm{Sgc} 8$ can be calculated as:

$$
F=\frac{[\mathrm{PTK} 7 \cdot \mathrm{Sgc} 8]}{[\mathrm{Sgc} 8]+[\mathrm{PTK} 7 \cdot \mathrm{Sgc} 8]+[\mathrm{SSB} \cdot \mathrm{Sgc} 8]}=\frac{\frac{[\mathrm{PTK} 7][\mathrm{Sgc} 8]}{\mathrm{Kd} 1}}{[\mathrm{Sgc} 8]+\frac{[\mathrm{PTK}][\mathrm{Sgc8} 8]}{\mathrm{Kd} 1}+\frac{[\mathrm{SSB}][\mathrm{Sgc8}]}{\mathrm{Kd} 2}}
$$

Divide both the top and the bottom by $\frac{[\mathrm{PTK} 7][\mathrm{Sgc} 8]}{\mathrm{Kd} 1}$

Which can also be expressed as:

$$
F=\frac{1}{\frac{\mathrm{Kd} 1}{[\mathrm{PTK} 7]}+1+\frac{[\mathrm{SSB}]}{\mathrm{Kd} 2} \cdot \frac{\mathrm{Kd} 1}{[\mathrm{PTK} 7]}}
$$

$$
F=\frac{1}{1+\frac{\mathrm{Kd} 1}{[\mathrm{PTK} 7]} \cdot\left(1+\frac{[\mathrm{SSB}]}{\mathrm{Kd} 2}\right)}
$$

Based on this equation, it should be noted that the ratio of $\frac{[\mathrm{PTK} 7 \cdot \mathrm{Sgc} 8]}{[\mathrm{Total} \operatorname{Sgc} 8]}$ and [SSB] are inversely related. The higher the concentration of SSB is, the lower the ratio would be.

$$
\begin{gathered}
\text { Total PTK7 }=[\text { PTK7 }]+[\text { PTK7 } \cdot \text { Sgc8 }] \\
{[\text { PTK7 }]<\text { Total PTK7 }}
\end{gathered}
$$

Therefore: 


$$
F=\frac{1}{1+\frac{\mathrm{Kd} 1}{[\mathrm{PTK} 7]} \cdot\left(1+\frac{[\mathrm{SSB}]}{\mathrm{Kd} 2}\right)}<\frac{1}{1+\frac{\mathrm{Kd} 1}{[\text { Total PTK7 }]} \cdot\left(1+\frac{[\mathrm{SSB}]}{\mathrm{Kd} 2}\right)}
$$

According to a previous study, the expression level of PTK7 on CCRF-CEM cells is $1300 \pm 190$ receptors $/ \mu \mathrm{m}^{2}$, the average diameter of this cell is $12 \pm 2 \mu \mathrm{m}$, and the cell density used in this study is $1.0 \times 10^{6} \mathrm{cell} / \mathrm{mL}$, the calculated total concentration of PTK 7 is around $1 \mathrm{nM}$ by assuming that the cell is nearly spherical. The total concentration of SSB used in this study is $10,000 \mathrm{nM}$, and there would only a small fraction of SSB $(0.01 \%$ at most $)$ would bind with Sgc8:

$$
\begin{gathered}
\text { Total SSB }=[\mathrm{SSB}]+[\mathrm{SSB} \cdot \mathrm{Sgc} 8] \\
{[\mathrm{SSB}] \gg[\mathrm{SSB} \cdot \mathrm{Sgc} 8]}
\end{gathered}
$$

Therefore:

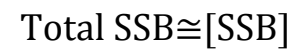

$F<0.0037$ Indicating that aptamer on cell surfaces almost completely displacement by SSB. 
Table S1. Sequences used in this work

\begin{tabular}{|c|c|c|c|}
\hline & $5^{\prime}$ & Sequences & $3^{\prime}$ \\
\hline Sgc8 & & $\begin{array}{l}\text { TCT AAC CGT ACA GTA TTT TCC } \\
\text { CGG CGG CGC AGC AGT TAG A }\end{array}$ & FITC \\
\hline Sgc8-DBCO & DBCO & $\begin{array}{l}\text { TCT AAC CGT ACA GTA TTT TCC } \\
\text { CGG CGG CGC AGC AGT TAG A }\end{array}$ & FITC \\
\hline RS-DBCO & DBCO & $\begin{array}{l}\text { ACC TAT AGG TGC TCT AGG TCA } \\
\text { CGC TAG CTT TCG TTT GTG TCC } \\
\text { TTG ATT CGT TGC ATC TAT A }\end{array}$ & FITC \\
\hline Lib & & $\begin{array}{l}\text { NNN NNN NNN NNN NNN NNN NNN } \\
\text { NNN NNN NNN NNN NNN }\end{array}$ & FITC \\
\hline $\begin{array}{l}\text { Sgc8c- } \\
\text { DBCO }\end{array}$ & DBCO & $\begin{array}{l}\text { TCT AAC CGT ACA GTA TTT TCC } \\
\text { CGG CGG CGC AGC AGT TAG A }\end{array}$ & TAMRA \\
\hline $\begin{array}{l}\text { TSgc8- } \\
\text { DBCO }\end{array}$ & DBCO & $\begin{array}{l}\text { CGT CCT TCA TAT CTA ACT GCT } \\
\text { GCG CCG CCG GGA AAA TAC TGT } \\
\text { ACG GTT AGA }\end{array}$ & FITC \\
\hline $\begin{array}{l}\text { Sgc8-6- } \\
\text { DBCO }\end{array}$ & DBCO & $\begin{array}{l}\text { AC CGT ACA GTA TTT TCC CGG } \\
\text { CGG CGC AGC AGT }\end{array}$ & FITC \\
\hline RS-DBCO & DBCO & $\begin{array}{l}\text { ACC TAT AGG TGC TCT AGG TCA } \\
\text { CGC TAG CTT TCG TTT GTG TCC } \\
\text { TTG ATT CGT TGC ATC TAT A }\end{array}$ & TAMRA \\
\hline Sgc8 & & $\begin{array}{l}\text { TCT AAC CGT ACA GTA TTT TCC } \\
\text { CGG CGG CGC AGC AGT TAG A }\end{array}$ & TAMRA \\
\hline RS & & $\begin{array}{l}\text { ACC TAT AGG TGC TCT AGG TCA } \\
\text { CGC TAG CTT TCG TTT GTG TCC } \\
\text { TTG ATT CGT TGC ATC TAT A }\end{array}$ & FITC \\
\hline $\begin{array}{l}\text { TSGCgc8- } \\
\text { CDNA }\end{array}$ & Dabcyl & $\begin{array}{l}\text { TCT AAC CGT ACA GTA TTT TCC } \\
\text { CGG CGG CGC AGC AGT TAG ATA } \\
\text { TGA AGG ACG }\end{array}$ & \\
\hline Sgc8-cDNA & Dabcyl & $\begin{array}{l}\text { ATC TAA CCG TAC AGT ATT TTC } \\
\text { CCG GCG GCG CAG CAG TTA GAT }\end{array}$ & \\
\hline $\begin{array}{l}\text { TSgc8- } \\
\text { CDNA }\end{array}$ & & $\begin{array}{l}\text { TCT AAC CGT ACA GTA TTT TCC } \\
\text { CGG CGG CGC AGC AGT TAG ATA } \\
\text { TGA AGG ACG }\end{array}$ & \\
\hline Sgc8-cDNA & & $\begin{array}{l}\text { ATC TAA CTG CTG CGC CGC CGG } \\
\text { GAA AAT ACT GTA CGG TTA GA }\end{array}$ & \\
\hline RS-cDNA & & $\begin{array}{l}\text { TAT AGA TGC AAC GAA TCA AGG } \\
\text { ACA CAA ACG AAA GCT AGC GTG } \\
\text { ACC TAG AGC ACC TAT AGG T }\end{array}$ & \\
\hline
\end{tabular}




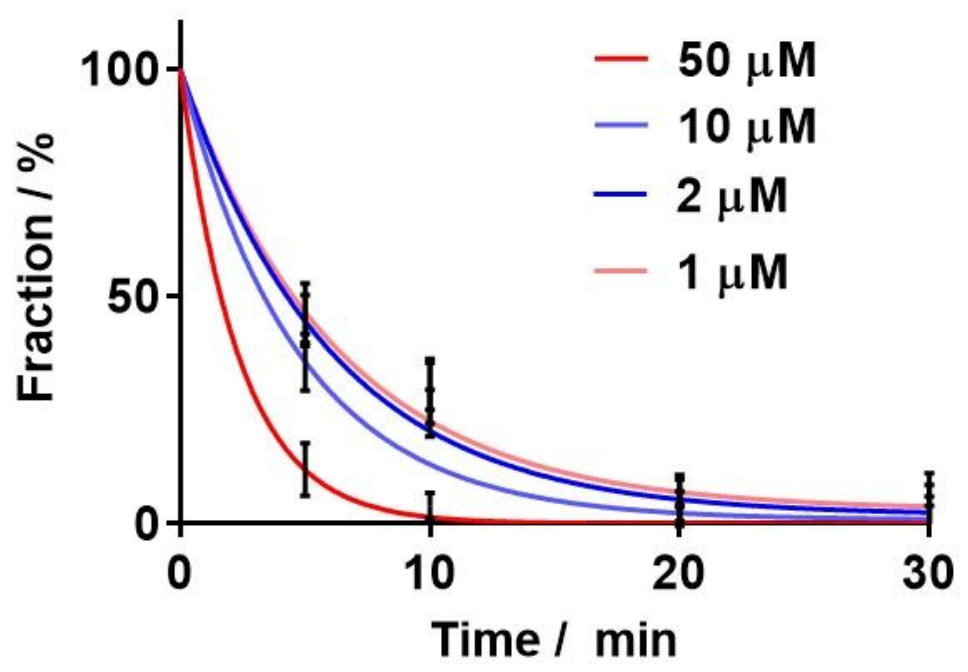

Figure S1. Evaluation of displacement kinetics of TSgc8 on CEM cells treated with $50,10,2$, and $1 \mu \mathrm{M}$ tcDNA at $37^{\circ} \mathrm{C}$ for 0.5 hour. $\left(\mathrm{K}_{\text {off }}=0.392,0.193\right.$, 0.153 , and $0.147 \mathrm{~min}^{-1}$ ) 

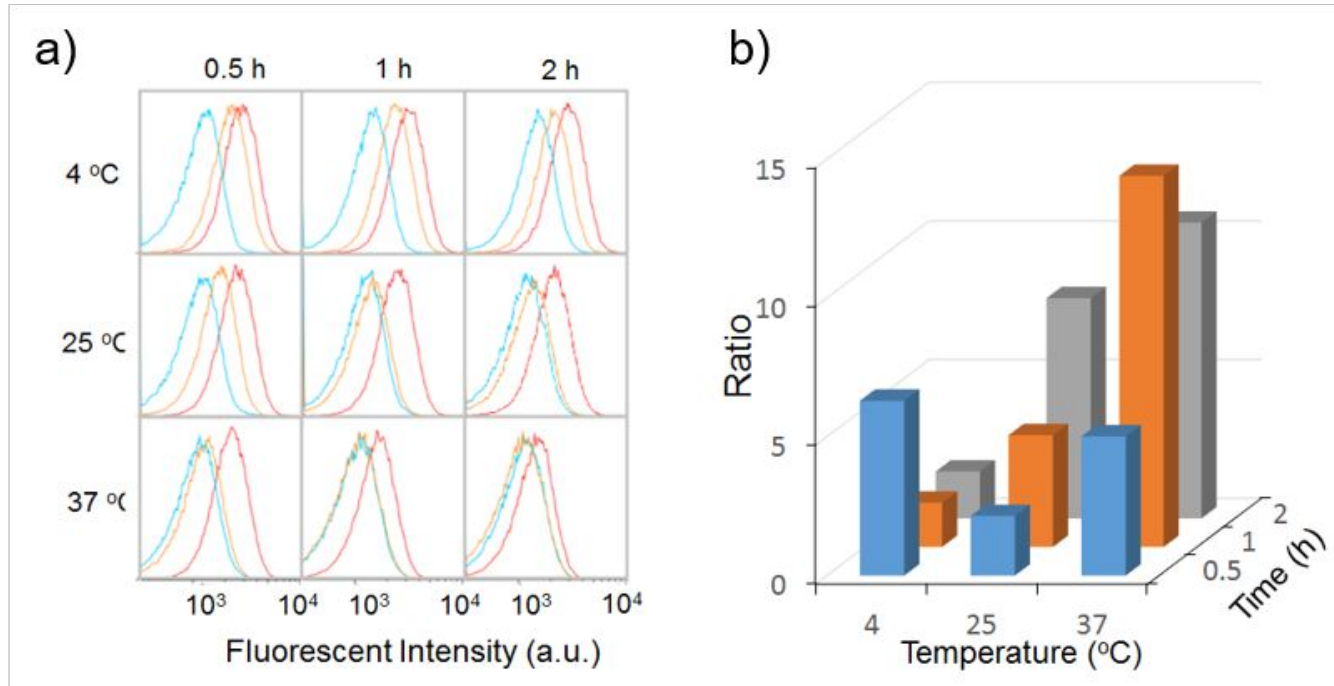

Figure S2. a) Incubation time and temperature effect on detaching aptamer from cell surface with cDNA. Red, yellow and blue lines represent the fluorescence intensities of cells treated with $500 \mathrm{nM}$ Sgc8-DBCO, Sgc8 and Lib, respectively, then incubated with $10 \mu \mathrm{M}$ cDNA in PBS for different time spans and temperatures. b) Graph showing signal to background ratio of a). 


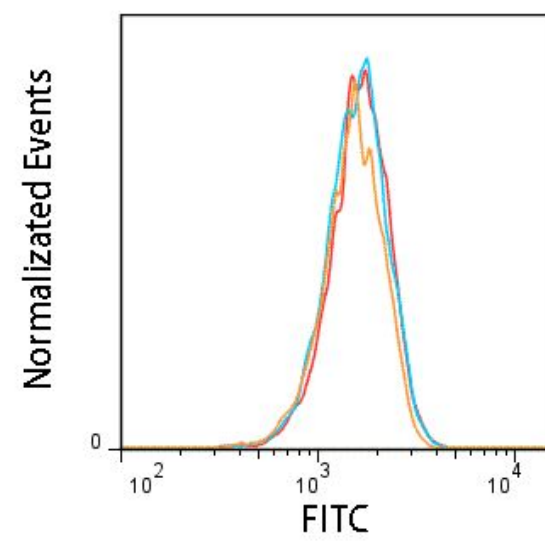

Non azide-bearing CEM cells
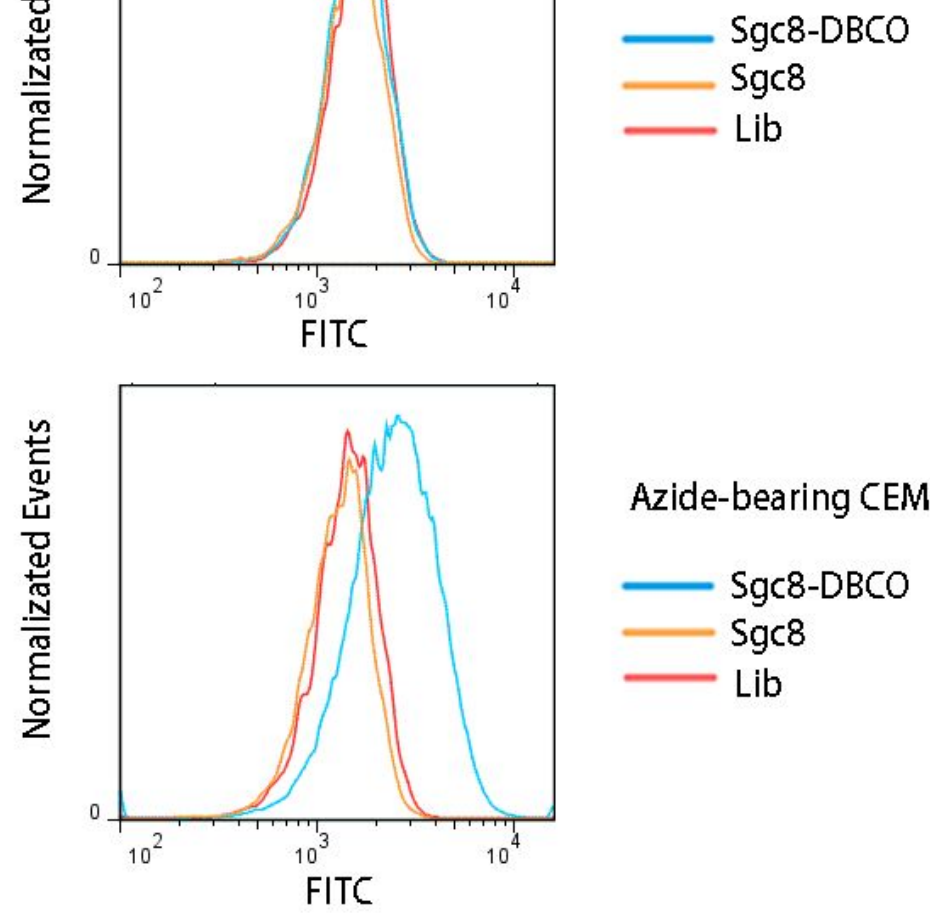

Azide-bearing CEM cells

- Sgc8-DBCO

Sgc8

$\longrightarrow$ Lib

Figure S3. Flow cytometric analysis of CEM cells cultured with/without Ac4ManNAz. Blue, orange and red lines represent the fluorescence intensity of cells treated with Sgc8-DBCO, Sgc8 and Lib, respectively. After washing with PBS buffer, Sgc8-DBCO and Sgc8 groups were treated with cDNA, following the standard procedure. The red lines show the background fluorescence intensity of cells with Lib. Our method can also be used to confirm the amount of nonspecific reaction. We applied FITC-labeled aptamer and FITC-aptamerDBCO conjugate to normal CEM cells without treatment with $\mathrm{Ac}_{4} \mathrm{ManNAz}$. After cDNA incubation, cells treated with Sgc8-DBCO had the same fluorescence signal as that of the Sgc8 and Lib groups. 

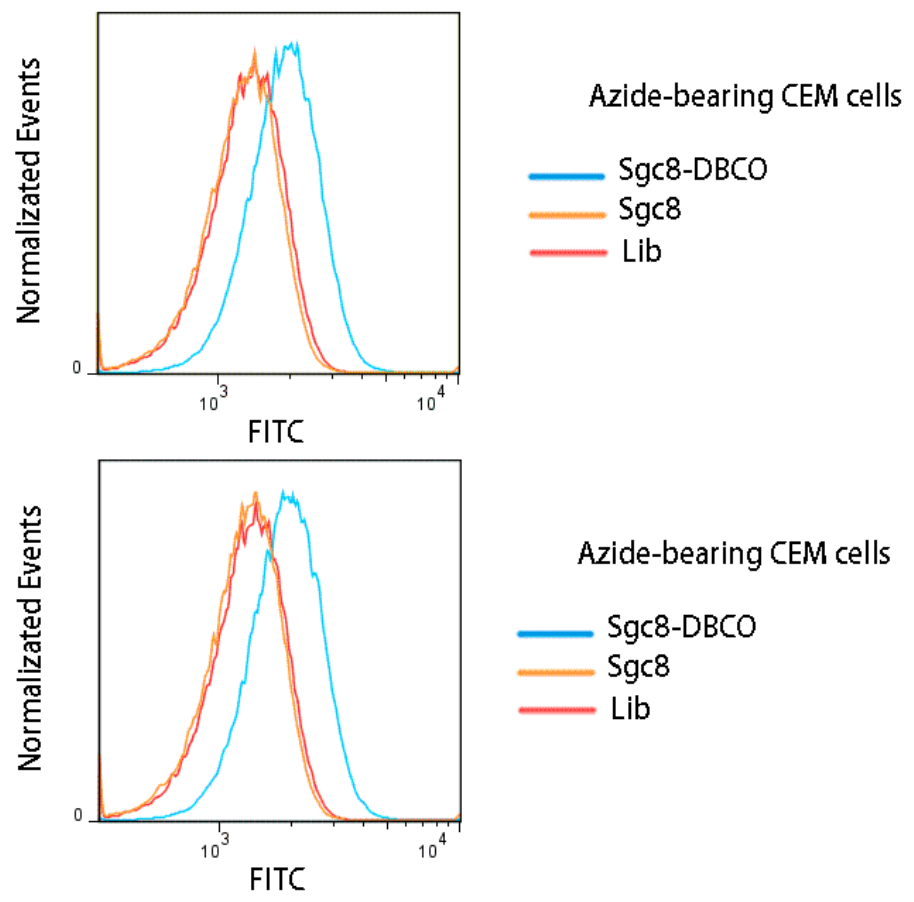

Figure S4. Flow cytometric analysis of glycoform detection using toehold mechanism (top) and cDNA (bottom). To shorten aptamer detachment time, regular aptamer was replaced with toehold aptamer conjugate, which showed comparable results, but in less time. 

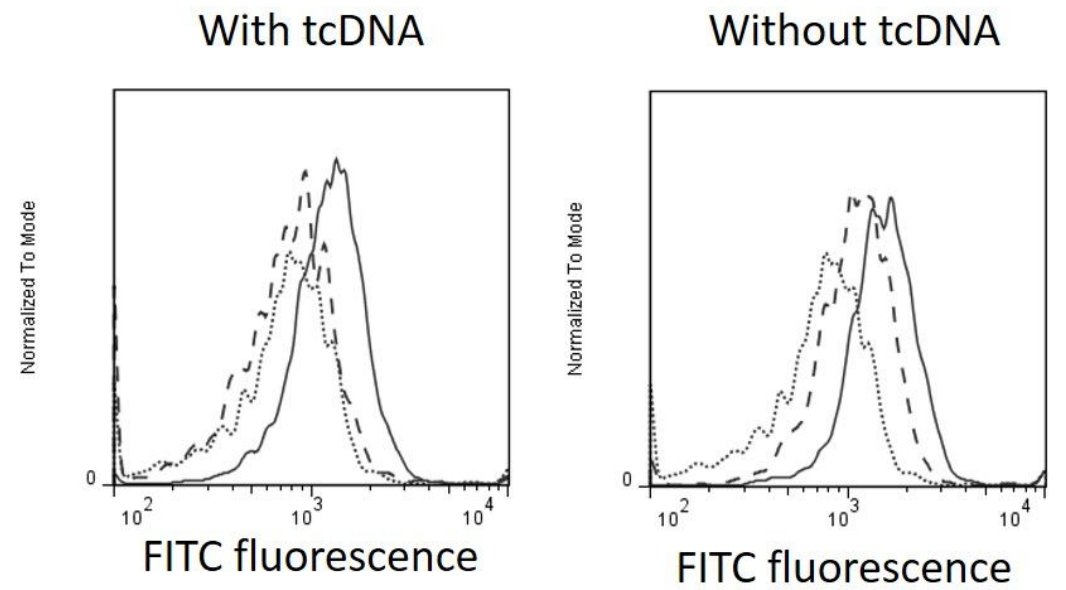

Figure S5. Flow cytometry assay of dissociation of Tsgc8 from CEM cells. Cells are incubated with TSgc8-DBCO(solid), Tsgc8(dashed) and Lib (library sequence) (dotted), washed, then incubation with PBS containing or not containing $10 \mu \mathrm{M}$ tcDNA at $37^{\circ} \mathrm{C}$. 
Table S2. The percentages and binding affinities of abundant sequences in SELEX pool.

\begin{tabular}{|c|c|c|c|}
\hline Name & Sequences & $\mathrm{Kd}(\mathrm{nM})$ & $\begin{array}{l}\text { Percetage } \\
\text { in pool }\end{array}$ \\
\hline L1 & $\begin{array}{c}\text { ATACCAGCTTATTCAATTCCACACGACTAATGCTACTAAACATGACGGAAGCCA } \\
\text { GATCCTTCACCGTGCGAGATAGTAAGTGCAATCT }\end{array}$ & $19 \pm 5$ & $0.05 \%$ \\
\hline L2 & $\begin{array}{l}\text { ATACCAGCTTATTCAATTCCACCAACAATAAATGAAAGGGACCAAGCATCACAT } \\
\text { ACCAACACACCCGGCGAGATAGTAAGTGCAATCT }\end{array}$ & $20 \pm 7$ & $0.06 \%$ \\
\hline L3 & $\begin{array}{l}\text { ATACCAGCTTATTCAATTCCACACAAACCAACAAACTTTCGGAATGTAGCGACA } \\
\text { CACCCCAGTCCCGGCGAGATAGTAAGTGCAATCT }\end{array}$ & $53 \pm 8$ & $0.05 \%$ \\
\hline L4 & $\begin{array}{l}\text { ATACCAGCTTATTCAATTCCACGATAAAGACAAACGCTATGAATGATCCTAACA } \\
\text { ACATCTCTCCGCTCGAGATAGTAAGTGCAATCT }\end{array}$ & $77 \pm 13$ & $0.06 \%$ \\
\hline L5 & $\begin{array}{l}\text { ATACCAGCTTATTCAATTCCAGACAGACCAATACAGATAAGAAACGACCATTCC } \\
\text { ATACTGTGACCGGCGAGATAGTAAGTGCAATCT }\end{array}$ & $78 \pm 11$ & $0.07 \%$ \\
\hline L6 & $\begin{array}{l}\text { ATACCAGCTTATTCAATTCCACGACAGAGAAGAGATCGACCCCAAAGAAATAC } \\
\text { GTCCTAAATGCCCGCCGAGATAGTAAGTGCAATCT }\end{array}$ & $97 \pm 8$ & $0.11 \%$ \\
\hline L7 & $\begin{array}{c}\text { ATACCAGCTTATTCAATTCCACGTGAGAGAACCGATTAATCCAGAATACACAAC } \\
\text { TCTACATGCCCCTCGAGATAGTAAGTGCAATCT }\end{array}$ & $134 \pm 10$ & $0.16 \%$ \\
\hline L8 & $\begin{array}{l}\text { ATACCAGCTTATTCAATTCCCCGACAATAATAGCGGCCCTAAAGATAATGTACA } \\
\text { TAGTTCCTGTCCGGCGAGATAGTAAGTGCAATCT }\end{array}$ & $241 \pm 26$ & $0.04 \%$ \\
\hline L9 & $\begin{array}{l}\text { ATACCAGCTTATTCAATTCCCACACATAGACAGACATCGAGAACAGAACATAG } \\
\text { GTTAACACACCCCGTCGAGATAGTAAGTGCAATCT }\end{array}$ & $835 \pm 57$ & $0.04 \%$ \\
\hline L10 & $\begin{array}{l}\text { ATACCAGCTTATTCAATTCCCACGACAATCACAATACAGTCCGGAGTGCACAAC } \\
\text { AAATAACCCCCTCTCGAGATAGTAAGTGCAATCT }\end{array}$ & $>1000$ & $0.04 \%$ \\
\hline L11 & $\begin{array}{l}\text { ATACCAGCTTATTCAATTCCACGGACAAGTCTTTATCACAATAATCTTACCTCTT } \\
\text { GCTCATTCCCGCTCGAGATAGTAAGTGCAATCT }\end{array}$ & $>1000$ & $0.13 \%$ \\
\hline L12 & $\begin{array}{l}\text { ATACCAGCTTATTCAATTCCACGGCGAAAGAACACTATTGATTATATGTCAGGC } \\
\text { ACCAAGTACCCCGGCGAGATAGTAAGTGCAATCT }\end{array}$ & $>1000$ & $0.10 \%$ \\
\hline L13 & $\begin{array}{l}\text { ATACCAGCTTATTCAATTCCCCGCAACAACCGACTACATGAACAGCCTAAGTCT } \\
\text { CCCAATTAATCCGTCGAGATAGTAAGTGCAATCT }\end{array}$ & $>1000$ & $0.05 \%$ \\
\hline L14 & $\begin{array}{l}\text { ATACCAGCTTATTCAATTCCCCGGCGCACAGTCATTAAACACAACCCAAAGTGT } \\
\text { GGTCTTCTCCCCCTCGAGATAGTAAGTGCAATCT }\end{array}$ & $>1000$ & $0.04 \%$ \\
\hline L15 & $\begin{array}{l}\text { ATACCAGCTTATTCAATTCCCACGATAACGAAATAAACTAACCTTCACTCTCTCT } \\
\text { TCAAATGTCCTGGCGAGATAGTAAGTGCAATCT }\end{array}$ & $>1000$ & $0.08 \%$ \\
\hline
\end{tabular}

